\title{
Enhanced hyperfine magnetic fields for face-centered tetragonal Fe(110) ultrathin films on vicinal $\operatorname{Pd}(110)$
}

\author{
B. Roldan Cuenya* \\ Physics Department, University of Central Florida, Orlando, Florida 32816, USA \\ W. Keune \\ Fachbereich Physik, Universität Duisburg-Essen (Campus Duisburg), D-47048 Duisburg, Germany \\ Dongqi Li and S. D. Bader \\ Materials Science Division, Argonne National Laboratory, Argonne, Illinois 60439, USA \\ (Received 5 October 2004; revised manuscript received 14 December 2004; published 18 February 2005)
}

\begin{abstract}
The structure and hyperfine magnetic properties of epitaxial Fe ultrathin films on a vicinal $\mathrm{Pd}(110)$ surface have been investigated by means of low-energy electron diffraction (LEED), reflection high-energy electron diffraction (RHEED) and ${ }^{57} \mathrm{Fe}$ conversion electron Mössbauer spectroscopy (CEMS). LEED and RHEED provide evidence for initial pseudomorphic film growth. The RHEED determination of the in-plane atomic distance versus Fe film thickness demonstrates the stabilization of the metastable fcc-like $\mathrm{Fe}$ structure on $\operatorname{Pd}(110)$. This interpretation is supported by in situ ${ }^{57} \mathrm{Fe}$ CEMS measurements which indicate an enhanced saturation hyperfine field of $\sim 39 \mathrm{~T}$ for a 3-monolayers-thick Fe film at $25 \mathrm{~K}$. This is the highest value ever measured for Fe on a metallic substrate. Our results suggest that ultrathin fcc-like (face-centered tetragonal) $\mathrm{Fe}$ films on $\operatorname{Pd}(110)$ are in a ferromagnetic high-moment state with an enhanced hyperfine field due to electronic $3 d-4 d$ hybridization at the $\mathrm{Fe} / \mathrm{Pd}$ interface.
\end{abstract}

DOI: 10.1103/PhysRevB.71.064409

PACS number(s): 75.75.+a, 68.65.-k, 76.80.+y

\section{INTRODUCTION}

Numerous experimental and theoretical investigations have shown that the magnetic properties of ultrathin magnetic layers supported on nonmagnetic substrates dramatically change when the dimensions of the system are reduced and the surface or interface symmetry is broken. ${ }^{1-4}$ Ultrathin epitaxially grown ferromagnetic (FM) layers are particularly interesting, as unusual new properties including perpendicular magnetic anisotropy, enhanced magnetic moments, and metastable phases have been observed. ${ }^{5-19}$ The present work reports enhanced hyperfine fields and discusses the structural and magnetic properties in the context of prior related research.

The magnetic behavior of the Fe/Pd system is of special interest due to its exotic exchange coupling. ${ }^{20-23}$ Rader $e t$ al. ${ }^{24,25}$ demonstrated that Pd becomes ferromagnetic when in proximity to Fe. This result is supported by several theoretical studies ${ }^{26,27}$ and giant $\mathrm{Pd}$ moments have been measured in dilute Fe-Pd alloys. ${ }^{28-30}$ Despite the large scientific effort dedicated in the last twenty years to the understanding of this system, many discrepancies regarding the magnitude of the Fe moment at the Pd interface and the origin of its reduction/ enhancement with respect to bulk bcc-Fe still remain. Hosoito et al. ${ }^{31}$ found by depth-profiling Mössbauer spectroscopy that $30 \%$ of the $\mathrm{Fe}$ atoms in a $3.5 \AA{ }^{57} \mathrm{Fe}$ probe layer at the Pd interface were paramagnetic at room temperature (RT) and had a reduced magnitude of the hyperfine field $\left(\left|B_{\mathrm{hf}}\right|\right)$ at $4.2 \mathrm{~K}$. They concluded that a tendency exists for Pd to quench magnetic order in $\mathrm{Fe}$ monolayers. Later on, Boufelfel et al. ${ }^{32}$ also reported a lower $\left|B_{\mathrm{hf}}\right|$ for interfacial Fe layers in $[\mathrm{Fe}(110) / \mathrm{Pd}(111)]_{n}$ superlattices with $n=1$, and slightly larger values than that of bulk bcc-Fe when thicker films were studied, but no paramagnetism was observed. ${ }^{32}$ This difference may be related to the structure of the $\mathrm{Fe}$ layers. Increased hyperfine fields were discovered by $\mathrm{Li}$ et $a l .{ }^{33}$ for interfacelike $\mathrm{Fe}$ in $\mathrm{Fe} / \mathrm{Pd}$ multilayers using Mössbauer spectroscopy. A change in texture was noted in that study, where the Pd layers were found to have fcc(111) texture, and the Fe layers bcc(110) texture, for Pd thicknesses $t_{\mathrm{Pd}}<36 \AA$, but fcc-Fe(111) texture for Pd $t_{\mathrm{Pd}} \geqslant 36 \AA$. More recently, Cheng et al. ${ }^{20}$ extracted a $14 \%$ enhancement in $\left|B_{\mathrm{hf}}\right|$ of the 2-ML-thick interfacial $\mathrm{Fe}$ region in magnetronsputtered $\mathrm{Fe} / \mathrm{Pd}$ multilayers by extrapolation of the temperature dependence of $\left|B_{\mathrm{hf}}\right|$ to low $T$. They assumed that the main contribution to the measured hyperfine field was due to the negative polarization of the core electrons (neglecting conduction electron polarization and dipole effects) and obtained a Fe magnetic moment of $2.8 \mu_{B}$. This value is in agreement with theoretical studies on $\mathrm{Fe} / \mathrm{Pd}(001)$ superlattices carried out by Stoeffler et al. ${ }^{27}$

We have used molecular beam epitaxy (MBE) in ultrahigh vacuum (UHV) for the controlled deposition of clean, epitaxial, ultrathin magnetic layers on a single crystal substrate. Among epitaxial $\mathrm{Fe} / \mathrm{Pd}, \mathrm{Fe}$ on $\mathrm{Pd}(100)$ is the most studied system experimentally ${ }^{34-38}$ and theoretically. ${ }^{39,40}$ This Pd orientation offers the smaller lattice mismatch $(3.3 \%)$ with respect to bcc-Fe, making it a desirable substrate for the pseudomorphic growth of ultrathin, crystalline, flat Fe films. Less attention has been devoted to other substrate orientations such as $\operatorname{Pd}(111)$, where the larger lattice mismatch with bcc-Fe (4.2\%) favors a strong tetragonalization of the epitaxial $\mathrm{Fe}$ layers and leads to a more complicated growth morphology. ${ }^{22,41}$ In addition, Fe films deposited on differ- 
ently oriented Pd surfaces also exhibit distinct magnetic properties. Fe films grown on $\operatorname{Pd}(100)$ display ferromagnetic (FM) behavior at RT above a critical thickness of 1.3-1.7 monolayers (ML). ${ }^{36} \mathrm{~A}$ minimum Fe thickness of 2-2.5 ML was reported for FM onset in the $\mathrm{Fe} / \mathrm{Pd}(111)$ system. ${ }^{22}$

The structure of ultrathin Fe films stabilized on differently orientated Pd surfaces and its correlation with their magnetic behavior have been intensively debated in the literature. Investigations carried out by Binns et al. ${ }^{41}$ on $\mathrm{Fe} / \mathrm{Pd}(111)$ using low-energy electron diffraction (LEED), auger electron spectroscopy (AES), and x-ray photoelectron spectrosocpy (XPS) pointed out that the first 2 ML of Fe grow with a facecentered cubic (fcc-)like structure and display antiferromagnetic (AF) order. For Fe coverages $>5 \mathrm{ML}$, local moments approaching bulk body-centered cubic (bcc-)Fe values were determined. Boeglin et al..$^{35}$ studied the growth and the interface of $\mathrm{Fe}$ films on $\mathrm{Pd}(100)$ by surface extended $\mathrm{x}$-ray absorption fine structure (SEXAFS) and observed a structural transition after 4 ML Fe from a face-centered tetragonal (fct) $\mathrm{Fe}_{55} \mathrm{Pd}_{45}$ alloy to body-centered tetragonal (bct) Fe layers. The large orbital moments measured for a 3-ML-thick Fe film were attributed to interface alloying and to the fct structure. On $[\mathrm{Fe} / \mathrm{Pd}]_{n}$ multilayers, Mühlbauer et al. ${ }^{42,43}$ also demonstrated by reflection high-energy electron diffraction (RHEED) the presence of a distorted fcc-like Fe-phase for Fe thicknesses $<25 \AA$. Magnetic susceptibility measurements conducted on these films lead to the report of $\mathrm{Fe}$ magnetic moments that are $20 \%$ larger than that in bulk bcc-Fe. This group related the appearance of the large moments observed to the presence of fcc-like Fe in a high-spin (HS) configuration rather than to polarization effects of $\mathrm{Pd}$ at the $\mathrm{Fe} / \mathrm{Pd}$ interfaces. ${ }^{42}$ Celinski et al. ${ }^{44}$ also found metastable fct $\mathrm{Fe}$ at the $\mathrm{Fe} / \mathrm{Pd}$ interface of ultrathin $\mathrm{Fe}(001) / \mathrm{Pd}(001) / \mathrm{Fe}(001)$ sandwich structures. The formation of two fcc-Fe phases with different lattice parameters in $e$-beam evaporated $\mathrm{Fe} / \mathrm{Pd}$ multilayers was recently reported by Pan et al., ${ }^{45}$ and a correlation between microstructure and magnetism was inferred. Other groups, however, reported the stabilization of interfacial ultrathin $\mathrm{Fe}$ films with bet structure on $\mathrm{Pd}(100),{ }^{46}$ on $\mathrm{Fe} / \mathrm{Pd}$ multilayers, ${ }^{20}$ and on $\mathrm{Pd}$ overlayers supported on $\operatorname{Ag}(001) .{ }^{47}$

Previous investigations on Fe films deposited on vicinal$\operatorname{Pd}(100)$ single crystals have demonstrated three points: (i) surface steps can induce an in-plane uniaxial magnetic anisotropy in the Fe overlayer, (ii) the easy axis is perpendicular to the step edges, and (iii) the strength of the anisotropy scales linearly with the step density. ${ }^{23}$ A similar dependence was observed for fcc-Co supported on vicinal- $\mathrm{Cu}(001){ }^{48}$ Other systems including bcc-Fe/vicinal- $\mathrm{Ag}(001)$ and bcc-Fe/ vicinal-W(001) displayed a quadratic relationship. ${ }^{49,50}$ The quadratic and linear dependencies arise from symmetry properties of the bcc and fcc lattices that are broken at the step edges. This offers the interesting opportunity of manipulating the magnetic anisotropy of a stepped thin film.

The Fe/vicinal-Pd system has been chosen for the present study for several reasons. First, the spin-orbit interaction of $\mathrm{Pd}$ always produces a strong magnetic anisotropy in FM/Pd systems, and, therefore, a strong step-induced, in-plane magnetic anisotropy can be expected. Second, Pd can be polarized at the $\mathrm{Fe} / \mathrm{Pd}$ interface and made to become FM. ${ }^{51}$ Choi et al. $^{23}$ demonstrated that $\mathrm{Fe}$ films supported on vicinal$\operatorname{Pd}(100)$ exhibit different magnetic properties compared to films deposited on flat surfaces. The Curie temperature of ultrathin step-supported Fe films $(<2 \mathrm{ML})$ is higher than that on flat surfaces, and an additional induced magnetic moment on Pd at the step edges appears. This sensitivity to the local environment of Pd arises from distinct electronic hybridization at different $\mathrm{Fe}$ coordination sites.

This paper reports on the epitaxial growth of ultrathin $\mathrm{Fe}$ films evaporated under UHV conditions on vicinal-Pd(110) substrates. The presence of an Fe-Pd surface alloy at the initial stage of growth will be discussed. The choice of this substrate orientation is motivated by literature reports indicating that the induced FM moment carried by $\mathrm{Pd}$ at the $\mathrm{Fe} / \mathrm{Pd}$ interface extends up to $\sim 5 \mathrm{ML}$ into the (110) surface of Pd. ${ }^{52}$ Combined RHEED, LEED and in situ ${ }^{57} \mathrm{Fe}$ CEMS measurements are used to prove that the high moment fct-Fe phase is stable at RT. The hyperfine (hf) field $\left(\left|B_{\mathrm{hf}}\right|\right.$ $=39.2 \mathrm{~T}$ ) for a $3-\mathrm{ML} \mathrm{Fe}$ film at $25 \mathrm{~K}$ is the highest ever measured for an Fe film on a metallic substrate.

\section{EXPERIMENTAL}

The experiments were carried out in an UHV chamber with base pressure $<6 \times 10^{-11}$ mbar equipped with LEED, RHEED, Auger and in situ ${ }^{57} \mathrm{Fe}$ CEMS. The pressure during film deposition $<2 \times 10^{-10}$ mbar. The $\operatorname{Pd}(110)$ substrate, ${ }^{53}$ with a vicinal angle of $5^{\circ} \pm 0.1^{\circ}$, was cleaned under UHV conditions by cycles of $\mathrm{Ar}^{+}$sputtering (ion energy: $0.5 \mathrm{keV}$ ) at $430{ }^{\circ} \mathrm{C}$ and annealing at $677^{\circ} \mathrm{C}$. The cleaned substrate and the subsequently deposited Fe films are free of any measurable contamination within Auger sensitivity.

The ${ }^{57} \mathrm{Fe}$ films (95.5\% isotopic enrichment) were deposited from Knudsen cells with alumina crucibles surrounded by water-cooled shrouds. The evaporation sources were outgassed and stabilized prior to sample deposition. The film thickness and deposition rate $(0.25 \AA \mathrm{A} / \mathrm{min})$ were measured by a quartz-crystal microbalance that was calibrated previously by RHEED intensity oscillations during fcc-Fe deposition onto a clean $\mathrm{Cu}(001)$ substrate. During deposition, the $\mathrm{Pd}$ substrate temperature was held at $70^{\circ} \mathrm{C}$.

The crystallographic order of the Pd crystal and Fe films were confirmed with RHEED and LEED, recorded on the fluorescence screen by using a charge-coupled device (CCD) camera. The diffraction images were analyzed digitally to quantify (i) the changes in the in-plane atomic spacing with increasing $\mathrm{Fe}$ thickness (via RHEED) as described previously ${ }^{54}$ and (ii) the average terrace size of the substrate (via LEED) prior to evaporation.

The ${ }^{57} \mathrm{Fe}$ CEM spectra were obtained in situ in UHV utilizing a channeltron electron detector placed in front of the sample and surrounded near its entrance cone by a $\mathrm{MgO}$ coated copper tube to convert part of the high-energy conversion electrons from the sample into low-energy secondary electrons from $\mathrm{MgO}$, and thus improving the efficiency of the channeltron. ${ }^{55}$ After preparation of the ${ }^{57} \mathrm{Fe}$ film the sample was transferred and fixed in the same UHV system to the cold finger of a liquid-helium flow cryostat. CEM spectra were measured in zero external field at $25 \mathrm{~K}$ (i.e., near mag- 

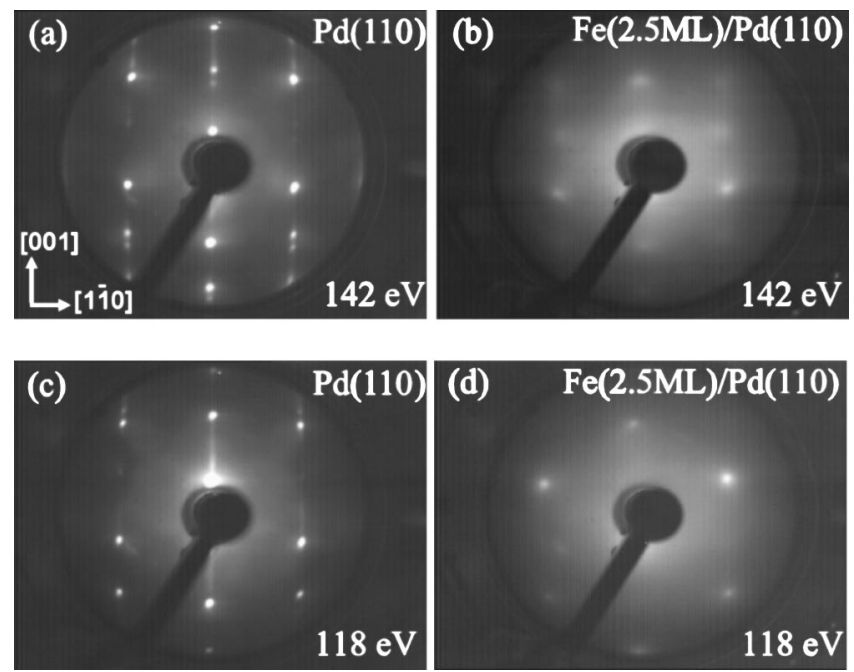

FIG. 1. LEED patterns (measured at room temperature) of the atomically clean Pd(110) vicinal surface (a),(c), and of 2.5 ML Fe epitaxially grown on vicinal $\operatorname{Pd}(110)$ (b),(d). All images have been taken under the same angular geometry keeping the distances of camera and sample to the LEED screen constant. The incident electron energies were $142 \mathrm{eV}$ (a),(c) and $118 \mathrm{eV}$ (b),(d).

netic saturation of $\mathrm{Fe}$ ) using a ${ }^{57} \mathrm{Co}$ Mössbauer source ( $\mathrm{Rh}$ matrix) of $\sim 40 \mathrm{mCi}$ activity placed outside the UHV system. The Mössbauer $\gamma$ radiation was transmitted through an UHVproof beryllium window in normal incidence to the sample plane. A Mössbauer drive system operating in constant acceleration mode combined with conventional electronics was employed.

\section{RESULTS AND DISCUSSION}

\section{A. LEED}

Figures 1(a) and 1(c) and display LEED images of the clean vicinal $\operatorname{Pd}(110)$ substrate at 142 and $118 \mathrm{eV}$, respectively. These LEED patterns were recorded a few degrees off from normal incidence. The bright central round spot clearly observable in Figs. 1(a) and 1(c) corresponds to the specular reflection or $(0,0)$ beam. The LEED patterns (a) and (c) show the superposition of sharp round spots with long streaks along the direction perpendicular to the step edges ([001]). Double spots, typical of an ordered vicinal surface with wide terraces, are also observed. From the distance between these double spots in Figs. 1(a) and 1(c), an average terrace size (w) of $\approx 18 \AA$ is estimated. This is consistent with the width of the terraces calculated for a $\operatorname{Pd}(110)$ surface with a vicinal angle of $5^{\circ}$ and $1.4-\AA$ atomic step height ${ }^{56}[w$ $\left.=(1.4 \AA) / \tan \left(5^{\circ}\right)=16 \AA\right]$.

Whereas the clean (110) surfaces of the late $5 d$ metals Ir, $\mathrm{Pt}$, and $\mathrm{Au}$ reconstruct spontaneously, ${ }^{57-60}$ it takes a high coverage of hydrogen (1 ML) to induce reconstruction on the (110) surface of the late $4 d \mathrm{Pd}^{61}$ Since the LEED patterns displayed in Fig. 1 were measured directly after substrate cleaning, we expect a minimum hydrogen coverage adsorbed on the $\mathrm{Pd}(110)$ surface and therefore no surface reconstruction.
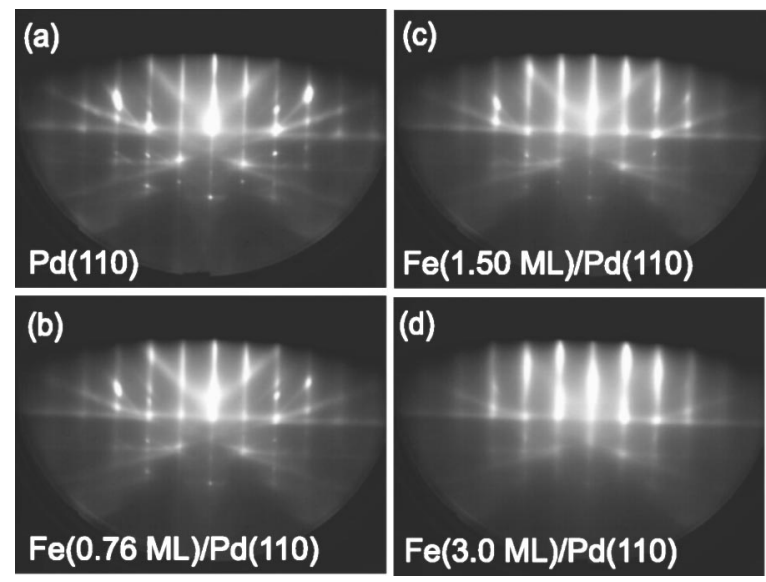

FIG. 2. RHEED patterns recorded at room temperature along the [001] azimuthal substrate direction with $10 \mathrm{keV}$ electron energy and $30 \mu \mathrm{A}$ beam current: (a) vicinal $\operatorname{Pd}(110)$ surface immediately prior to evaporation; and after deposition of $0.76 \mathrm{ML} F e(b), 1.50$ ML Fe (c), and 3.0 ML Fe (d). All images have been taken under the same angular geometry keeping the distances of camera and sample to the RHEED screen constant.

After the deposition of 2.5 ML Fe [see Figs. 1(b) and $1(d)$, blurred, round spots arranged in a rectangular substratelike cell become visible. The large background intensity observed compared to that for clean $\mathrm{Pd}(110)$ qualitatively corresponds to small ordered domains superimposed on disordered areas. The LEED pattern indicates pseudomorphic Fe growth and the stabilization of a compressed fcc-like (fct) Fe film, as will be demonstrated by means of RHEED. The disappearance of the double reflections that were present in Figs. 1(a) and 1(c) points to a full coverage of the substrate surface, in accordance with an initial layer-by-layer growth mode, as previously observed on this system. ${ }^{18}$

\section{B. RHEED}

RHEED measurements were carried out with the $10-\mathrm{keV}$, $30-\mu \mathrm{A}$ electron beam along the $[001]$ of the $\operatorname{Pd}(110)$ surface. For the clean surface [Fig. 2(a)] and Fe films $<1.5 \mathrm{ML}$ [Figs. 2(b) and 2(c)], sharp streaks are observed, indicating smooth, well-ordered surfaces. The first Fe layer grows pseudomorphically with the same lattice parameter as the $\mathrm{Pd}$ substrate. Above $\sim 1.6 \mathrm{ML}$ Fe [Fig. 2(d)], the streaks become broad, indicating the onset of 3D growth.

Since the distance between the streaks is inversely proportional to the in-plane atomic spacing, a precise determination of the in-plane atomic distance (perpendicular to the scattering plane) relative to that of the $\operatorname{Pd}(110)$ substrate can be carried out from the analysis of the RHEED patterns. ${ }^{54}$ The result is plotted in Fig. 3 .

After the deposition of the first 0.5-ML Fe, the in-plane atomic spacing decreases by $\sim 0.6 \%$. Between 0.5 and about $1.3 \mathrm{ML}$, a plateaulike behavior is observed, with only $\sim 0.4 \%$ decrease between 0.5 and $1 \mathrm{ML}$, followed by $\sim 0.7 \%$ decrease between 1-1.3 ML. Thus, the films grow pseudomorphically in this thickness range, with a maximum compression of $\sim 1.7 \%$ compared to fcc- $\operatorname{Pd}(110)$ and an in-plane 


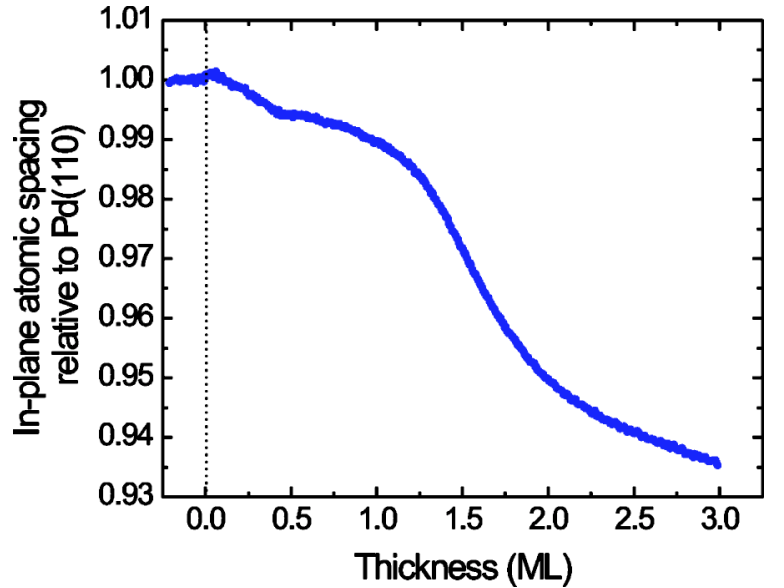

FIG. 3. Thickness dependence of the Fe in-plane atomic distance relative to that of $\operatorname{Pd}(110)$ obtained from RHEED at room temperature. (The electron beam was along the azimuthal [001] substrate direction.)

nearest-neighbor (NN) distance of $2.7 \AA$. This value is close to the value of $2.69 \AA$ for the $\mathrm{Fe}-\mathrm{Pd} \mathrm{NN}$ distance in a distorted-fcc $\mathrm{Fe}_{50} \mathrm{Pd}_{50}$ alloy. ${ }^{35}$ Studies performed by Boeglin et al. ${ }^{35}$ on $\mathrm{Fe} / \mathrm{Pd}(100)$ also showed the formation of an Fe-Pd surface alloy with an in-plane compressed and out-of-plane extended fct structure similar to the tetragonalized $\gamma$ phase of the disordered $\mathrm{Fe}_{x} \mathrm{Pd}_{1-x}$ alloy. ${ }^{62}$

Above 1.3 ML Fe the in-plane atomic distance is reduced more rapidly with increasing film thickness (Fig. 3), providing evidence of structural relaxation of the Fe films. This is consistent with the observed 3D growth that starts above 1.5 ML, as shown in Fig. 2. The in-plane atomic spacing approaches saturation at $\sim 3 \mathrm{ML}$ with a total compression of $\sim 7 \%$.

If one tentatively assumes that in the initial stage of growth the epitaxy is for bcc $\mathrm{Fe}(110)$ to match the fcc $\operatorname{Pd}(110)$ surface [Figs. 4(a) and 4(b)], then unrelaxed pseudomorphic bcc Fe should experience an in-plane compression (a)

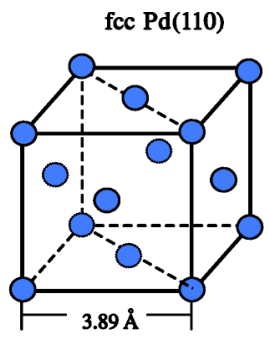

(b)

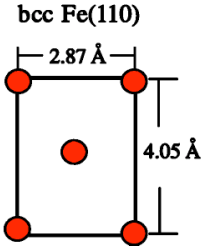

fcc $\operatorname{Pd}(110)$

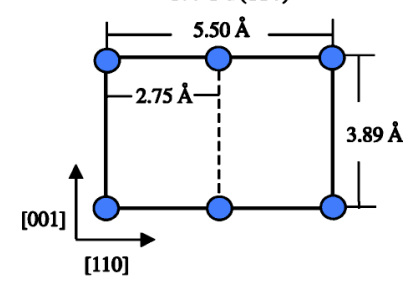

(c)

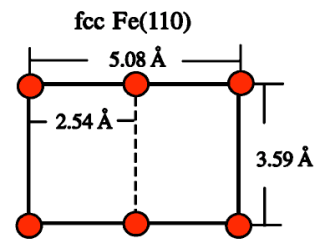

FIG. 4. Schematics of (a) fcc-Pd(110), (b) bcc-Fe(110), and (c) fcc-Fe(110) surface structures. The lattice parameters are 3.89, 2.87, and $3.59 \AA$ for $\mathrm{Pd}$, bcc $\mathrm{Fe}$, and fcc $\mathrm{Fe}$, respectively, at room temperature. of $\sim 4.2 \%$ according to Fig. 4 . Therefore, with increasing Fe thickness, a relaxation towards the larger bulk-Fe atomic spacing should occur, which disagrees with our experimental data. (We measured a $\sim 7 \%$ compression of the in-plane atomic distance.)

Another possibility is that the initial growth creates expanded fcc-like Fe [Fig. 4(c)]. Indeed, a plot of the lattice parameter of (high-moment) ferromagnetic and (lowmoment) antiferromagnetic (AFM) fcc Fe alloys versus the number of electrons per atom $(e / a)$ extrapolated to metastable pure fcc $\mathrm{Fe}(e / a=8)$ leads to a lattice parameter of $a_{0}=3.64 \AA$ (for FM high-moment fcc Fe) and $a_{0}=3.58 \AA$ (for AFM low-moment fcc Fe). ${ }^{63}$ These values are 6.4 and $8 \%$, respectively, smaller than $a_{0}=3.89 \AA$ of bulk Pd. Thus, an in-plane expanded fcc-like phase in the initial stage of growth followed by a reduction of the expansion at larger $\mathrm{Fe}$ coverages (up to $3 \mathrm{ML}$ ) would be consistent with the observed lattice compression with increasing Fe thickness (Fig. 3 ), though it would involve a much larger lattice mismatch ( $\sim 6.4$ to $\sim 8 \%$ expansion) with respect to the Pd(110) surface than for bcc $\mathrm{Fe}(110)$ ( $\sim 4.2 \%$ compression). The existence of fcc-like Fe is consistent with the fact that according to our LEED (Fig. 1) and RHEED (Fig. 2) patterns the initial growth is pseudomorphic.

Tetragonal lattice distortions were previously observed by Boeglin et al. ${ }^{35}$ during the RT epitaxial growth of $\mathrm{Fe}$ on $\operatorname{Pd}(100)$. Analysis of LEED patterns using the $I(V)$ method lead to out-of-plane lattice parameters very close to that of bulk fcc-Pd for Fe thicknesses lower than 4 ML (3.90 $)$ ). With increasing thickness, a rapid reduction of the out-ofplane lattice spacing down to $3.1 \AA$ was observed and a structural transition from a tetragonal distorted fcc to a bcc structure was inferred. Additional chemical information from SEXAFS (Ref. 35) supports the idea of a structural transition from an fct Fe-Pd alloy (mixed interface) to bct-Fe with increasing $\mathrm{Fe}$ thickness. Another study of $\mathrm{Fe}$ growth on $\operatorname{Pd}(001)$ suggests that above $10 \mathrm{ML}$ the epitaxial Fe films are body-centered tetragonal $;{ }^{38}$ however, these researchers could not fit their LEED $I / V$ results at lower $\mathrm{Fe}$ coverage $^{38}$ to this model.

The present LEED and RHEED results provide evidence for the fcc-like (fct-type) Fe structure on $\operatorname{Pd}(110)$ in the initial stages of growth. It will be shown in the next section that our CEMS results on $3 \mathrm{ML} \mathrm{Fe} / \mathrm{Pd}(110)$ are consistent with the result provided by the structural investigation.

\section{C. ${ }^{57} \mathrm{Fe}$ CEMS}

The CEM spectra displayed in Figs. 5(a) and 5(b) were recorded immediately after RT deposition of 3 and $8 \mathrm{ML} \mathrm{Fe}$, respectively, on the vicinal $\operatorname{Pd}(110)$ single crystal and after cooling to $25 \mathrm{~K} .{ }^{57} \mathrm{Fe}$ CEM measurements provide isotopespecific information about the structure, local environment, magnetic anisotropy and approximate magnitude of the $\mathrm{Fe}$ magnetic moment of the ultrathin ${ }^{57} \mathrm{Fe}$ films.

Both spectra displayed in Figs. 5(a) and 5(b) exhibit a nuclear Zeeman sextet, indicating large hyperfine magnetic field values $B_{\mathrm{hf}}$. The films are magnetically ordered at $25 \mathrm{~K}$. Because of the rather large apparent width of the outer lines 


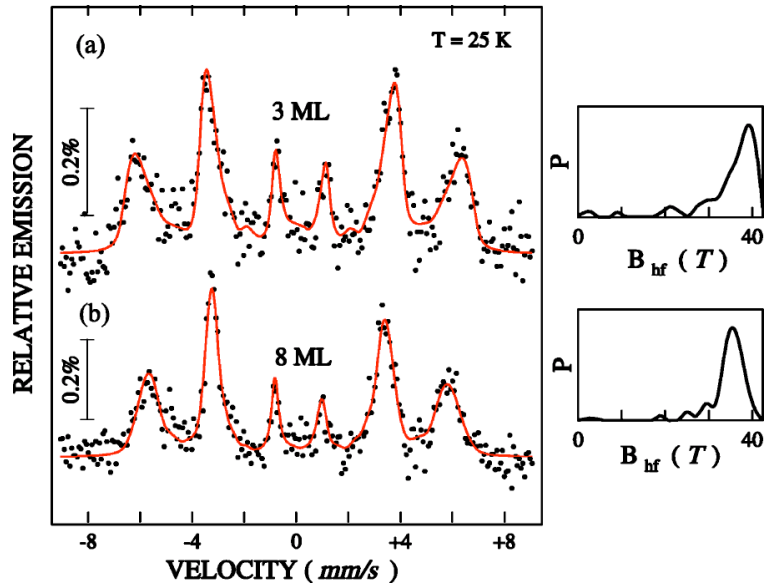

FIG. 5. Mössbauer spectra (CEMS) of $3 \mathrm{ML}{ }^{57} \mathrm{Fe}$ (a) and $8 \mathrm{ML}$ ${ }^{57} \mathrm{Fe}(\mathrm{b})$ on vicinal $\mathrm{Pd}(110)$ measured in situ in UHV at $25 \mathrm{~K}$. Right-hand side: distribution of hyperfine magnetic fields $P\left(B_{\mathrm{hf}}\right)$.

and the spectral asymmetry observed, the spectra have been least-squares fitted with a distribution of hyperfine magnetic fields $P\left(B_{\mathrm{hf}}\right)$, including a weak mean electric-quadrupole nuclear level shift $\epsilon{ }^{64}$ The Mössbauer spectral parameters obtained from the fitting are given in Table I, together with experimental results for 3-4-ML-thick epitaxial fct-Fe layers deposited on $\mathrm{Cu}_{3} \mathrm{Au}(001)$ (Ref. 17) and $\mathrm{Cu}(001),{ }^{65,66}$ and UHV grown polycrystalline 15 - $\AA$-thick Fe layers in $\mathrm{Fe} / \mathrm{Pd}$ multilayers. ${ }^{67}$

The spectrum of 3 ML Fe [Fig. 5(a)] and its Mössbauer parameters $\left(B_{\mathrm{hf}}\right.$ and $2 \epsilon$, Table I) are distinctly different from those of bulk bcc-Fe $\left(\left|B_{\mathrm{hf}}\right|=34.0 \mathrm{~T}, \delta=+0.12 \mathrm{~mm} / \mathrm{s},{ }^{68} 2 \epsilon\right.$ $=0 \mathrm{~mm} / \mathrm{s}$ at low temperature), and rather close to those of fct-Fe/ $/ \mathrm{Cu}_{3} \mathrm{Au}(001),{ }^{17}$ fct-Fe/Cu(001), ${ }^{65,66}$ or fct-Fe in $\mathrm{Fe} / \mathrm{Pd}$ multilayers. ${ }^{67}$ The fit indicates a hyperfine field distribution with a peak at $39.2 \mathrm{~T}$ [Fig. 5(a)], which not only is very large compared to the value of $34.0 \mathrm{~T}$ measured at $25 \mathrm{~K}$ for bulk bcc-Fe, but is the largest $\left|B_{\mathrm{hf}}\right|$ value ever observed for $\mathrm{Fe}$ films on metallic substrates. The 8-ML Fe film deposited on $\operatorname{Pd}(110)$ [Fig. 5(b)] also shows an enhanced hyperfine field (with a peak value of $35.2 \mathrm{~T}$, Table I) relative to that of bulk bcc-Fe, but one that is much smaller than that measured for the 3-ML-thick Fe film.
Since the electric quadrupole interaction arises from the presence of an electric-field gradient (EFG) at the ${ }^{57} \mathrm{Fe}$ nucleus, the nuclear level shift $2 \epsilon$ is related to the degree of asymmetry in the local electronic distribution. The observed relatively large (negative) quadrupole level shift $(2 \epsilon)$ of $-0.09 \mathrm{~mm} / \mathrm{s}$ for 3-ML $\mathrm{Fe} / \mathrm{Pd}(110)$ provides a modelindependent proof for the locally noncubic structure (lattice distortion) of the 3-ML Fe film. For bulk Fe with ideal bcc structure $2 \epsilon$ is zero. The quadrupole level shift $2 \epsilon$ obtained for the 8-ML Fe film is negligible within error bars, pointing towards a much less distorted lattice of this Fe film. By analogy with SEXAFS results ${ }^{35}$ on 7-ML-thick Fe films on $\operatorname{Pd}(001)$ we may conclude that our 8-ML film on Pd(110) has bct structure.

The average center-line shift $\delta$ at $25 \mathrm{~K}$ given in Table I includes the chemical shift (isomer shift) and the temperature-dependent, second-order Doppler shift (thermal redshift). The positive shifts obtained for the 3-ML ( $\delta$ $=0.22 \mathrm{~mm} / \mathrm{s})$ and $8-\mathrm{ML}(\delta=0.16 \mathrm{~mm} / \mathrm{s})$ samples as compared to the shift of bulk bcc-Fe at $25 \mathrm{~K}[\delta=0.12 \mathrm{~mm} / \mathrm{s}$ (Ref. 68)] indicate a decrease of the $s$-electron density at the ${ }^{57} \mathrm{Fe}$ nucleus in the films as compared to bulk bcc-Fe. This means that $s$-like electrons of the interfacial Fe regions are transferred to Pd. Similar results $(\delta=0.15 \mathrm{~mm} / \mathrm{s})$ were attained by $\mathrm{Li}$ et $a l .{ }^{33}$ for interfacial Fe layers in [fcc(111)$\mathrm{Fe}(20 \AA) / \mathrm{fcc}(111)-\operatorname{Pd}(36 \AA)]_{25}$ multilayers. Our result is also in agreement with the theory by Miedema and Van der Woude ${ }^{69}$ for the concentration (c) dependence of isomer shift values in binary $\mathrm{Fe}_{c} X_{(1-c)}$ alloys that predicts interatomic electron transfer to the more electronegative element, in this case Pd (Pauling electronegativity of $\mathrm{Pd}=\chi_{\mathrm{Pd}}=2.20$ versus $\left.\chi_{\mathrm{Fe}}=1.83\right)$.

Direct information about the average Fe spin orientation in zero external field [given by the angle $\theta$ between the incident $\gamma$-ray direction (or film normal direction) and the direction of $B_{\mathrm{hf}}$ (i.e., the $\mathrm{Fe}$ spin direction)] is obtained from the line intensity ratios $3: R: 1: 1: R: 3$ of a Zeeman sextet, where $R$ is related to $\theta$ by

$$
\theta=\arccos \sqrt{\frac{4-R}{4+R}}
$$

From the least-squares fit of the CEM spectrum in Fig. 5(a) we obtain the ratio $I_{2,5} / I_{3,4}=R=3.5 \pm 0.2$, which yields

TABLE I. Mössbauer spectral parameters obtained from least-squares fitting of the CEM spectra in Fig. 5: center-line shift $\delta$ (including the isomer shift and second-order Doppler shift) relative to bulk bcc-Fe at room temperature, electric-quadrupole nuclear level shift $2 \epsilon$, average angle $\theta$ between the $\gamma$-ray direction and the Fe spin direction, and magnitude of the average hyperfine magnetic field $B_{\mathrm{hf}}$. Also given is the magnitude of the most-probable hyperfine magnetic field (peak). Mössbauer spectral parameters for ultrathin fct-Fe films on $\mathrm{Cu}_{3} \mathrm{Au}(001)$ (Ref. 17), $\mathrm{Cu}(001)$ (Refs. 65 and 66), and in UHV-deposited polycrystalline Fe/Pd multilayers (Ref. 67) are also shown for comparison. $T_{m}$ is the measurement temperature.

\begin{tabular}{lccccc}
\hline \hline Sample & $T_{m}(\mathrm{~K})$ & $\delta(\mathrm{mm} / \mathrm{s})$ & $2 \epsilon(\mathrm{mm} / \mathrm{s})$ & $\theta\left(^{\circ}\right)$ & $B_{\mathrm{hf}}(\mathrm{T})$ \\
\hline${ }^{57} \mathrm{Fe}(3 \mathrm{ML}) / \mathrm{Pd}(110)$ & $25 \mathrm{~K}$ & $0.217 \pm 0.002$ & $-0.09 \pm 0.03$ & 75 & $34.5 \pm 0.4$ (peak) 39.2 \\
${ }^{57} \mathrm{Fe}(8 \mathrm{ML}) / \mathrm{Pd}(110)$ & $25 \mathrm{~K}$ & $0.16 \pm 0.08$ & $-0.01 \pm 0.02$ & 90 & $34.2 \pm 0.4$ (peak) 35.2 \\
${ }^{57} \mathrm{Fe}(4 \mathrm{ML}) / \mathrm{Cu} \mathrm{Au}_{3}(001)($ Ref. 17) & $25 \mathrm{~K}$ & $0.26 \pm 0.08$ & $0.07 \pm 0.02$ & 25 & $36.2 \pm 0.5$ \\
{$\left[\mathrm{Fe}(15 \AA / \mathrm{Pd}(40 \AA)]_{30} /\right.$ Sapphire (Ref. 67) } & $4.2 \mathrm{~K}$ & $0.180 \pm 0.002$ & $-0.01 \pm 0.01$ & 79 & $35.5 \pm 0.3$ (peak) 36.8 \\
${ }^{57} \mathrm{Fe}(3 \mathrm{ML}) / \mathrm{Cu}(001)($ Ref. 66) & $56 \mathrm{~K}$ & & $-0.10 \pm 0.01$ & 80 & $32 \pm 1$ \\
\hline \hline
\end{tabular}


$\theta=75^{\circ} \pm 4^{\circ}$ for the $3-\mathrm{ML} \mathrm{Fe} / \mathrm{Pd}(110)$. This indicates a preferred in-plane spin orientation, analogous to the case of 6-ML Fe $(15 \AA)$ in Fe/Pd multilayers, ${ }^{67}$ where $\theta \sim 79^{\circ}$. An in-plane orientation of the magnetization is obtained also for the $8-\mathrm{ML} \mathrm{Fe} / \mathrm{Pd}(110)$ sample by fitting the spectrum of Fig. 5(b), where $R=4$ and $\theta=90^{\circ}$.

Under the assumptions that the main component of the EFG $V_{z z}$ is oriented approximately along the film normal direction and the asymmetry parameter $\eta$ is about equal to zero (local axial symmetry), the angle between $V_{z z}$ and the local spin direction is equal to the angle $\theta$ between the film normal direction and the incident $\gamma$-ray direction. Then, $2 \epsilon$ is given by $e Q V_{z z}\left(3 \cos ^{2} \theta-1\right) / 4 .^{64}$ (The nuclear quadrupole moment $Q$ of ${ }^{57} \mathrm{Fe}$ is $0.16 \times 10^{-24} \mathrm{~cm}^{2}$, see Ref. 70.) The measured values of $2 \epsilon=-0.09 \mathrm{~mm} / \mathrm{s}$ and $\cos ^{2} \theta=0.0670$ (or $\theta=75^{\circ}$ ) for $3-\mathrm{ML} \mathrm{Fe} / \mathrm{Pd}(110)$ then lead to a value for the quadrupole-interaction constant $e Q V_{z z} / 4$ of $+0.11 \mathrm{~mm} / \mathrm{s}$ (or $+5.4 \times 10^{-9} \mathrm{eV}$ ) or (equivalently) to a value for $V_{z z}$ of +1.4 $\times 10^{17} \mathrm{~V} / \mathrm{cm}^{2}$. These values are in good agreement with the corresponding quadrupole-interaction constant ${ }^{65}$ and $V_{z z}$ value $^{66}$ observed in ferromagnetic 3 -ML-thick distorted fcc (fct) Fe films on $\mathrm{Cu}(001)$ substrates. Apparently the degree of local lattice distortion of 3-ML-thick fct Fe films does not depend sensitively on the type of substrate used, but rather is an intrinsic property of the ferromagnetic fct structure. The change in sign of $2 \epsilon$ (Table I) in going from $\mathrm{Fe}(3 \mathrm{ML}) /$ $\mathrm{Pd}(110)$ to $\mathrm{Fe}(4 \mathrm{ML}) / \mathrm{Cu}_{3} \mathrm{Au}(001)$ is explained by the different $\mathrm{Fe}$ spin orientations in both systems: nearly in-plane on $\mathrm{Pd}(110)$, and preferentially perpendicular orientation on $\mathrm{Cu}_{3} \mathrm{Au}(001)$.

The strong $15 \%$ enhancement (relative to bulk bcc Fe at $25 \mathrm{~K}$ ) in magnitude of the measured most-probable (peak-) hyperfine field $B_{\mathrm{hf}}$ in $\mathrm{Fe}(3 \mathrm{ML}) / \mathrm{Pd}(110)$ cannot be explained by the additional contribution of the demagnetizing field, ${ }^{71}$ $\left|B_{\text {dem }}\right| \simeq \mu_{0} M_{S} \cos \theta$, for the case of nearly in-plane spin orientation. In our case, the external field is zero, and $|B|_{\text {dem }}$ $\simeq \mu_{0} M_{S} \cos \theta \simeq 0.5 \mathrm{~T}$ (taking $\theta=75^{\circ}$ and assuming that fct and bcc Fe have about the same saturation magnetization $\mu_{0} M_{S}$ of about $2 \mathrm{~T}$ ), which is very small. Therefore, the magnitude of the measured hyperfine field $\left(\left|B_{\mathrm{hf}}\right|\right)$ is about equal to the magnitude of the intrinsic hyperfine field $\left(\left|B_{\text {int }}\right|\right.$ $\left.=\left|B_{\mathrm{hf}}\right|-\left|B_{\mathrm{dem}}\right|=38.5 \mathrm{~T} \approx\left|B_{\mathrm{hf}}\right|\right)$. We may pose the question whether this observed enhanced $B_{\text {int }}$ is correlated with an enhancement in the Fe atomic moment $\mu_{\mathrm{Fe}}$ in the ultrathin film. [It is well known that in bulk Fe alloys there is roughly a proportionality between $B_{\text {int }}$ and $\mu_{\mathrm{Fe}}$, with a conversion factor of about $15 \mathrm{~T} / \mu_{B}$ (Ref. 72).] In fact, an enhanced moment of $\mu_{\mathrm{Fe}}=2.7 \mu_{B}$ was deduced by Mühlbauer et al. ${ }^{42}$ from magnetization measurements on polycrystalline $\mathrm{Fe} / \mathrm{Pd}$ multilayers, and the value of $2.67 \mu_{B}$ was inferred by Le Cann et al. ${ }^{37}$ from magnetic circular dichroism measurements on $\mathrm{Fe}(3 \mathrm{ML}) / \mathrm{Pd}(001)$. Assuming that no induced polarization occurred at the $\mathrm{Fe} / \mathrm{Pd}$ interface, polarized-neutron reflectometry results by Bland et al. ${ }^{47}$ also provided average $\mathrm{Fe}$ atomic moments of $2.66 \mu_{B}$ for $\mathrm{Pd} / \mathrm{Fe}(5.6 \mathrm{ML}) / \mathrm{Ag}(001)$ structures. The same value $\left(2.66 \mu_{B}\right)$ was observed by Fullerton et al. ${ }^{73}$ on $\mathrm{Au} / \mathrm{Pd} / \mathrm{Fe}(5.6 \mathrm{ML}) / \mathrm{Ag}(001)$. We will discuss the question of proportionality of $\left|B_{\mathrm{hf}}\right|$ and $\mu_{\mathrm{Fe}}$ further below.

The hyperfine field enhancement observed for the distorted fcc-like 3-ML Fe film on $\operatorname{Pd}(110)$ is not likely due to the additional induced moment of $\mathrm{Pd}$ at the step edges. But rather it is due to the increased $\mathrm{Fe}$ atomic moment associated with the metastable ferromagnetic, tetragonally distorted fcc (fct) Fe structure. This hypothesis is supported by the work of Mühlbauer et al. ${ }^{42}$ who observed a correlation between the enhanced Fe atomic moment $\left(2.7 \mu_{B}\right)$ and the fcc Fe structure in Fe/Pd multilayers. However, the finding by Li et al. ${ }^{33}$ of almost identical hyperfine magnetic fields (at RT) for fcc-Fe and bcc-Fe in the Fe/Pd multilayers with different Pd thicknesses are at variance with our experimental observations, since the structural transition that we observe in single-layer Fe films with increasing Fe thickness is accompanied by changes in $B_{\mathrm{hf}}$.

The possibility of Fe-Pd intermixing during the RT evaporation of ultrathin $\mathrm{Fe}$ films on $\mathrm{Pd}$ single crystals and in epitaxially grown Fe-Pd multilayers has been suggested previously by some authors, ${ }^{22,32}$ but excluded by others. ${ }^{24,25}$ An upper limit of three atomic layers was set for the amount of interdiffusion using x-ray diffraction data. ${ }^{32}$ Before one can attribute the enhanced hyperfine field value measured by CEMS on $\mathrm{Fe}(3 \mathrm{ML}) / \mathrm{Pd}(110)$ to high-moment fcc-like Fe, the effect of $\mathrm{Fe}-\mathrm{Pd}$ interfacial alloying or Fe interdiffusion into the Pd crystal should be considered.

Fe-Pd bulk alloys are ferromagnetic at RT for Fe concentrations down to 12 at. \%. ${ }^{74}$ If interdiffusion leads to $\mathrm{Fe}-\mathrm{Pd}$ alloy formation with $\mathrm{Fe}$ concentrations below this value, then the alloy will be paramagnetic at RT. ${ }^{74}$ Mössbauer studies carried out at RT by Boufelfel et al. ${ }^{32}$ on $[\mathrm{Fe}(4.2 \AA) / \mathrm{Pd}]_{n}$ superlattices with $n<10$ reveal a reduced distribution of $B_{\mathrm{hf}}$ values with a maximum at $32 \mathrm{~T}$ and a shoulder at $25 \mathrm{~T}$ for the first atomic layer of $\mathrm{Fe}$ in contact with $\mathrm{Pd}$. No paramagnetism was observed, however. These results are in agreement with RT Mössbauer measurements conducted on Pdrich $\mathrm{Pd}-\mathrm{Fe}$ solid solutions where reduced hyperfine fields were measured. ${ }^{21}$ Investigations by Boeglin et al. ${ }^{35}$ on $\mathrm{Fe} /$ $\mathrm{Pd}(001)$ provided evidence for the formation of an $\mathrm{Fe}-\mathrm{Pd}$ surface alloy with similar properties to the disordered $\gamma$ phase of $\mathrm{Fe}_{50} \mathrm{Pd}_{50}$. Using SEXAFS, they established a direct relationship between the formation of an interfacial alloy with tetragonal fct structure and the large orbital moments of $\mathrm{Fe}$ atoms in the $\mathrm{Fe}(3 \mathrm{ML}) / \mathrm{Pd}(001)$ system. An early survey of the magnetic properties of transition metals alloyed with Fe reported increased hyperfine fields for the Fe-Pd system. ${ }^{75}$ Van der Woude and Sawatzky ${ }^{76}$ also mention a hyperfine field increase of $1.1 \mathrm{~T}$ for an Fe-Pd alloy with respect to bcc-Fe. Klimars et al. ${ }^{77}$ measured enhanced values of $B_{\mathrm{hf}}$ for Fe-rich Fe-Pd alloys by Mössbauer spectroscopy. A maximum hyperfine field of $\sim 37.5 \mathrm{~T}$ at $20 \mathrm{~K}$ was measured for fcc-Fe ${ }_{1-x} \mathrm{Pd}_{x}$ alloys with $x \leqslant 0.37$. Increasing $\mathrm{Pd}$ concentration leads to a reduction of $\left|B_{\mathrm{hf}}\right|\left[\left|B_{\mathrm{hf}}\right|=35 \mathrm{~T}\right.$ for $\mathrm{Fe}_{0.5} \mathrm{Pd}_{0.5}$ (Ref. 78)]. These results were also qualitatively corroborated by Zhang et al. ${ }^{62}$ although smaller hyperfine fields were measured for the Fe-rich Fe-Pd alloys $(\sim 35 \mathrm{~T})$. However, the large hyperfine field value of $\sim 39 \mathrm{~T}$ that we measured for the $\mathrm{Fe}(3 \mathrm{ML}) / \mathrm{Pd}(110)$ sample cannot be explained exclusively by taking into consideration the formation of an interfacial Fe-Pd alloy.

We propose that an additional mechanism (in addition to interface alloying, if at all) is responsible for the large $\mathrm{hf}$ value of $\sim 39 \mathrm{~T}$ observed in the $3 \mathrm{ML}$ fct Fe film. Such a 
mechanism could be hybridization of $4 d-\mathrm{Pd}$ and $3 d$-Fe electronic wave functions in the interfacial $\mathrm{Fe} / \mathrm{Pd}$ region, combined with the two-dimensional character of the 3-ML-thick Fe film, as inferred from spin-resolved ab initio electronic band structure calculations. ${ }^{15,24,27,79}$ In this context it is worth mentioning that in a depth selective CEMS interface study of Pd coated epitaxial bcc-Fe(001) film structures the magnitude of $B_{\mathrm{hf}}$ was found to oscillate with the distance from the interface. ${ }^{80}$ Even at RT $B_{\mathrm{hf}}$ was found to be enhanced to a value of $\sim 37.8 \mathrm{~T}$ in the second $\mathrm{Fe}$ monolayer from the interface, and approached the bcc-Fe bulk value within 8-10 Fe monolayers. This oscillating behavior was explained by a superposition of an exponential short-range exchange interaction (mainly due to $3 d-4 d$ hybridization ${ }^{80}$ ) within the first three Fe monolayers from the interface, and an oscillating RKKY-type long-range exchange interaction (via conduction electrons) in deeper Fe layers. A similar hybridization effect at the fct-Fe/Pd(110) interface may lead to the strongly enhanced $B_{\mathrm{hf}}$ value observed in the present work.

Finally we would like to address the question of proportionality between the intrinsic hyperfine field $B_{\text {int }}$ and the $\mathrm{Fe}$ atomic moment $\mu_{\mathrm{Fe}}$ at and near the $\mathrm{Fe} / \mathrm{Pd}$ interface. It is well established that the measured (negative) hyperfine field at the ${ }^{57} \mathrm{Fe}$ nucleus $B_{\mathrm{hf}}$ can be decomposed as

$$
\begin{aligned}
B_{\mathrm{hf}} & =B_{\text {int }}+B_{\mathrm{dem}}=B_{\text {core }}+B_{\text {val,core }}+B_{\mathrm{val}, \mathrm{tr}}+B_{\mathrm{dem}} \\
& =B_{\mathrm{hf}, \mathrm{loc}}+B_{\mathrm{val}, \mathrm{tr}}+B_{\mathrm{dem}} .
\end{aligned}
$$

The small (positive) demagnetizing field $B_{\text {dem }}$ is estimated to be $<0.5 \mathrm{~T}$ in our case (as mentioned above) and will be neglected. $B_{\text {core }}$ and $B_{\text {val,core }}$ are the (negative) contributions due to intra-atomic polarization of $(1 s, 2 s, 3 s)$ Fe core electrons and $4 s$ valence electrons, respectively, by the local $3 d$ moment of the Fe Mössbauer atom, while $B_{\text {val,tr }}$ is the transferred hyperfine field due to inter-atomic polarization of valence $4 s$ electrons by $d$ electrons of neighboring $\mathrm{Fe}$ (and $\mathrm{Pd}$ ) atoms. While $B_{\mathrm{hf}, \text { loc }}=B_{\text {core }}+B_{\text {val,core }}$ is proportional to the local $3 d$ moment $^{81,82}\left(\mu_{3 d} \sim \mu_{\mathrm{Fe}}\right), B_{\text {val,tr }}$ depends on the degree of hybridization of valence $4 s$ electrons with $d$ electrons of neighboring atoms surrounding the Mössbauer atom, and on their magnetic moments. Hence, $B_{\text {val,tr }}$ is of nonlocal nature.

In their pioneering theoretical work Ohnishi et al. ${ }^{81,82}$ have demonstrated for the ground-state magnetism of ideally flat bcc-Fe(001) surfaces/interfaces that $B_{\text {val,tr }}$ changes sign from positive directly at the surface/interface (atomic layer $S$ ) to negative in the first subsurface/subinterface (layer $S$ -1 ) and following layers (layers $S-i, i \geqslant 2$ ). This results in a nontrivial relationship between $B_{\text {int }}$ and $\mu_{\mathrm{Fe}}$ in layers $S$ and $S-1$ of the ideally flat bcc-Fe(001) surface/interface.

For the case of the $\mathrm{Pd} / \mathrm{bcc}-\mathrm{Fe}(001)$ system with an ideally flat interface, Handschuh and Blügel ${ }^{79}$ have determined the monolayer-resolved ground-state Fe magnetic moments and ${ }^{57} \mathrm{Fe}$ hyperfine fields from $a b$ initio full-potential linearized augmented plane wave (FLAPW) calculations. They found an interfacial $\mathrm{Fe}$ region of about four atomic layers (layers $S$, $S-1, S-2, S-3)$, where $\mu_{\mathrm{Fe}}$ decreases monotonically from an enhanced value of $\sim 2.75 \mu_{B}$ (at layer $S$ ) to $\sim 2.3 \mu_{B}$ (at layer $S-1$ ) to its bulk value of $\sim 2.12 \mu_{B}$ (at layer $S-3$ ). Simultaneously, the magnitude of the hyperfine field $\left|B_{\text {int }}\right|$ was found to be nonmonotonic (peak-shaped) with depth, exhibiting strong enhancement with a maximum value of $\sim 38.5 \mathrm{~T}$ in the first subinterface layer (layer $S-1$ ), and a lower value (though still enhanced relative to the bulk value of $34.3 \mathrm{~T}$ ) of $\sim 35.8 \mathrm{~T}$ at the $\mathrm{Pd} / \mathrm{Fe}$ interface (layer $S$ ). The computed maximum value of $38.5 \mathrm{~T}$ is in excellent agreement with our experimentally observed peak value of $\left|B_{\text {int }}\right|$ $=39.2 \mathrm{~T}$ or with our value of $\left|B_{\text {int }}\right|=38.5 \mathrm{~T}$ after correcting with $B_{\text {dem }}$, although the crystallographic structure and orientation differ for theory $[\mathrm{Pd} / \mathrm{bcc}-\mathrm{Fe}(001)]$ and experiment [vicinal fct-Fe/Pd(110)], and ideally flat interfaces were assumed in the calculations. Although some Fe-Pd interdiffusion in our samples cannot be completely excluded (as discussed above), the good agreement between theory and experiment, on the other hand, suggests that such interdiffusion is nearly negligible in our samples, in accordance with Refs. 24, 25, and 80.

The theoretical results by Handschuh and Blügel ${ }^{79}$ demonstrate that, due to a change in sign of $B_{\text {val,tr, the }}$ monolayer-resolved proportionality between $\left|B_{\text {int }}\right|$ and $\mu_{\mathrm{Fe}}$ is lost at the interface (layer $S$ ) and at the first subinterface layer $(S-1)$ of the $\mathrm{Pd} / \mathrm{bcc}-\mathrm{Fe}(001)$ system. It is important to notice, however, that due to the peculiarity of $B_{\text {val,tr }}$ the average hyperfine field $\left\langle B_{\mathrm{hf}}\right\rangle_{3} \mathrm{ML}$ and the average Fe atomic moment $\left\langle\mu_{B}\right\rangle_{3 \mathrm{ML}}$, both averaged over a 3-ML-thick surface region, yield values of $36.2 \mathrm{~T}$ and $2.4 \mu_{B}$, respectively, according to theory. This results in a theoretical conversion factor $\left\langle B_{\mathrm{hf}}\right\rangle_{3 \mathrm{ML}} /\left\langle\mu_{B}\right\rangle_{3 \mathrm{ML}}$ of $15.1 \mathrm{~T} / \mu_{B}$, which agrees surprisingly well with the usual conversion factor of $15 \mathrm{~T} / \mu_{B}$ for bulk bcc-Fe alloys. ${ }^{72}$ This observation provides some justification for the inference of an enhanced average Fe magnetic moment $\left\langle\mu_{B}\right\rangle_{3 \mathrm{ML}}$ of $\sim 2.3 \mu_{B}$ from our measured average hyperfine field $\left\langle B_{\mathrm{hf}}\right\rangle_{3 \mathrm{ML}}$ of $34.5 \mathrm{~T}$ within our 3-ML-thick Fe film. The value of $2.3 \mu_{B}$ (and $34.5 \mathrm{~T}$ ) should be considered as a lower limit, because the hyperfine field distribution in Fig. 5 shows a low-field tail which might be due to a small fraction of thermally rapidly relaxing $\mathrm{Fe}$ spins in the ultrathin film at $25 \mathrm{~K}$.

\section{CONCLUSIONS}

The structure and hyperfine magnetic properties of epitaxial Fe ultrathin films on a vicinal Pd(110) surface have been investigated by means of LEED, RHEED, and in situ ${ }^{57} \mathrm{Fe}$ CEMS. LEED and RHEED provide evidence for pseudomorphic film growth in the initial stage of growth. The RHEED determination of the in-plane atomic distance versus Fe film thickness demonstrates the initial stabilization of the metastable fcc-like (fct) Fe structure on Pd(110). This interpretation is supported by ${ }^{57} \mathrm{Fe}$ CEMS measurements in ultrahigh vacuum which indicate an enhanced saturation hyperfine field of $\sim 39 \mathrm{~T}$ for a 3-ML-thick Fe film at $25 \mathrm{~K}$. A large, though less enhanced hyperfine field is also observed in distorted fcc-like (fct) Fe films on other substrates, such as $\mathrm{Cu}_{3} \mathrm{Au}(001) \cdot{ }^{17}$ These results suggest that ultrathin fcc-like Fe films on $\operatorname{Pd}(110)$ are in a ferromagnetic high-moment state, and show an enhanced $\mathrm{Fe}$ magnetic moment due to electronic $3 d-4 d$ hybridization at the $\mathrm{Fe} / \mathrm{Pd}$ interface. 


\section{ACKNOWLEDGMENTS}

We are grateful to U. von Hörsten (Duisburg) for valuable technical assistance and to S. Blügel (Jülich) for providing unpublished data and for valuable discussions. This work was supported by the Deutsche Forschungsgemeinschaft (GRK 277 and SFB 491) and at Argonne by the USDOEBES/MS under Contract No. W-31-109-ENG-38.
*Email address: roldan@ @hysics.ucf.edu

${ }^{1}$ U. Gradmann, T. Duerkop, and H. J. Elmers, J. Magn. Magn. Mater. 165, 56 (1997).

${ }^{2}$ U. Gradmann, J. Magn. Magn. Mater. 100, 481 (1991).

${ }^{3}$ S. D. Bader, Surf. Sci. 500, 172 (2002).

${ }^{4}$ R. Wu and A. J. Freeman, J. Magn. Magn. Mater. 200, 498 (1999).

${ }^{5}$ Utrathin Magnetic Structures I, edited by J. A. C. Bland and B. Heinrich (Springer, Berlin, 1994).

${ }^{6}$ G. A. Prinz, J. Magn. Magn. Mater. 100, 469 (1991).

${ }^{7}$ B. T. Jonker, K.-H. Walker, E. Kisker, G. A. Prinz, and C. Carbone, Phys. Rev. Lett. 57, 142 (1986).

${ }^{8}$ N. C. Koon, B. T. Jonker, F. A. Volkening, J. J. Krebs, and G. A. Prinz, Phys. Rev. Lett. 59, 2463 (1987).

${ }^{9}$ C. Liu, E. R. Moog, and S. D. Bader, Phys. Rev. Lett. 60, 2422 (1988).

${ }^{10}$ J. G. Tobin, G. D. Waddill, and D. P. Pappas, Phys. Rev. Lett. 68, 3642 (1992).

${ }^{11}$ J. Thomassen, F. May, B. Feldmann, M. Wuttig, and H. Ibach, Phys. Rev. Lett. 69, 3831 (1992).

${ }^{12}$ D. Li, M. Freitag, J. Pearson, Z. Q. Qiu, and S. D. Bader, Phys. Rev. Lett. 72, 3112 (1994).

${ }^{13}$ S. Müller, P. Bayer, C. Reischl, K. Heinz, B. Feldmann, H. Zillgen, and M. Wuttig, Phys. Rev. Lett. 74, 765 (1995).

${ }^{14}$ M. Zharnikov, A. Dittschar, W. Kuch, C. M. Schneider, and J. Kirschner, Phys. Rev. Lett. 76, 4620 (1996).

${ }^{15}$ C. L. Fu and A. J. Freeman, Phys. Rev. B 35, 925 (1987).

${ }^{16}$ R. E. Camley and D. Li, Phys. Rev. Lett. 84, 4709 (2000).

${ }^{17}$ B. Roldan Cuenya, M. Doi, S. Löbus, R. Courths, and W. Keune, Surf. Sci. 493, 338 (2001).

${ }^{18}$ Dongqi Li, B. Roldan Cuenya, J. Pearson, S. D. Bader, and W. Keune, Phys. Rev. B 64, 144410 (2001).

${ }^{19}$ A. Biedermann, R. Tscheliessnig, M. Schmid, and P. Varga, Phys. Rev. Lett. 87, 086103 (2001).

${ }^{20}$ L. Cheng, Z. Altounian, D. H. Ryan, and J. O. Ström-Olsen, J. Appl. Phys. 91, 7188 (2002).

${ }^{21}$ G. C. Papaefthymiou, K. J. Bryden, and J. Y. Ying, Physica B 311, 279 (2002).

${ }^{22}$ J. H. Choi, T. U. Nahm, Wookje Kim, Wondong Kim, J. Chung, J. Y. Kim, H. Koh, and S. J. Oh, Surf. Sci. 495, 173 (2001).

${ }^{23}$ H. J. Choi, R. K. Kawakami, E. J. Escorcia-Aparicio, Z. Q. Qiu, J. Pearson, J. S. Jiang, Dongqi Li, and S. D. Bader, Phys. Rev. Lett. 82, 1947 (1999).

${ }^{24}$ O. Rader, C. Carbone, W. Clemens, E. Vescovo, S. Blügel, W. Eberhardt, and W. Gudat, Phys. Rev. B 45, 13823 (1992).

${ }^{25}$ O. Rader, E. Vescovo, J. Redinger, S. Blügel, C. Carbone, W. Eberhardt, and W. Gudat, Phys. Rev. Lett. 72, 2247 (1994).

${ }^{26}$ A. B. Klautau, P. R. Peduto, and S. Frota-Pessoa, J. Magn. Magn. Mater. 186, 223 (1998).

${ }^{27}$ D. Stoeffler, K. Ounadjela, J. Sticht, and F. Gautier, Phys. Rev. B 49, 299 (1994).
${ }^{28}$ R. P. Peters, Ch. Buchal, M. Kubota, R. M. Mueller, and F. Pobell, Phys. Rev. Lett. 53, 1108 (1984).

${ }^{29}$ J. Crangle, Philos. Mag. 5, 335 (1960).

${ }^{30}$ R. M. Bozorth, P. A. Wolff, D. D. Davis, V. B. Compton, and J. H. Wernick, Phys. Rev. 122, 1157 (1961).

${ }^{31}$ N. Hosoito, T. Shinjo, and T. Takada, J. Phys. Soc. Jpn. 50, 1903 (1981).

${ }^{32}$ A. Boufelfel, R. M. Emrick, and C. M. Falco, Phys. Rev. B 43, 13152 (1991).

${ }^{33}$ M. Li, X. D. Ma, C. B. Peng, J. G. Zhao, L. M. Mei, Y. H. Liu, B. G. Shen, and D. S. Dai, J. Phys.: Condens. Matter 6, L785 (1994).

${ }^{34}$ C. Liu and S. D. Bader, J. Vac. Sci. Technol. A 8, 2727 (1990).

${ }^{35}$ C. Boeglin, H. Bulou, J. Hommet, X. Le Cann, H. Magnan, P. Le Fevre, and D. Chandesris, Phys. Rev. B 60, 4220 (1999).

${ }^{36}$ X. F. Jin, J. Barthel, J. Shen, S. S. Manoharan, and J. Kirschner, Phys. Rev. B 60, 11809 (1999).

${ }^{37}$ X. Le Cann, C. Boeglin, B. Carriere, and K. Hricovini, Phys. Rev. B 54, 373 (1996).

${ }^{38}$ J. Quinn, Y. S. Li, H. Li, D. Tian, and F. Jona, Phys. Rev. B 43, 3959 (1991).

${ }^{39}$ C. Li, A. J. Freeman, H. J. F. Jansen, and C. L. Fu, Phys. Rev. B 42, 5433 (1990).

${ }^{40}$ S. Blügel, B. Drittler, R. Zeller, and P. H. Dederichs, Appl. Phys. A: Solids Surf. 49, 547 (1989).

${ }^{41}$ C. Binns, C. Norris, G. P. Williams, M. G. Barthes, and H. A. Padmore, Phys. Rev. B 34, 8221 (1986).

${ }^{42}$ H. Mühlbauer, Ch. Müller, and G. Dumpich, J. Magn. Magn. Mater. 192, 423 (1999).

${ }^{43}$ T. Steffl, B. Rellinghaus, H. Mühlbauer, Ch. Müller, H. Herper, and G. Dumpich, in Recent Developments in Oxide and Metal Epitaxy-Theory and Experiment, edited by M. Yeadon et al., Mater. Res. Soc. Symp. Proc. No. 619 (Materials Research Society, Warrendale, PA, 2000), p. 79.

${ }^{44}$ Z. Celinski, B. Heinrich, J. F. Cochran, W. B. Muir, and A. S. Arrott, Phys. Rev. Lett. 65, 1156 (1990).

${ }^{45}$ F. Pan and Z. S. Zhang, Physica B 293, 237 (2001).

${ }^{46}$ C. Liu and S. D. Bader, J. Appl. Phys. 67, 5758 (1990).

${ }^{47}$ J. A. C. Bland, C. Daboo, B. Heinrich, Z. Celinski, and R. D. Bateson, Phys. Rev. B 51, 258 (1995).

${ }^{48}$ R. K. Kawakami, M. O. Bowen, H. J. Choi, E. J. EscorciaAparicio, and Z. Q. Qiu, Phys. Rev. B 58, R5924 (1998).

${ }^{49}$ J. H. Choi, Z. Q. Qiu, J. Pearson, S. J. Jiang, D. Li, and S. D. Bader, Phys. Rev. B 57, R12 713 (1998).

${ }^{50}$ R. K. Kawakami, E. J. Escorcia-Aparicio, and Z. Q. Qiu, Phys. Rev. Lett. 77, 2570 (1996).

${ }^{51}$ Jan Vogel, A. Fontaine, V. Cros, and F. Petroff, Phys. Rev. B 55, 3663 (1997).

${ }^{52}$ W. Weber, D. A. Wesner, D. Hartmann, and G. Günterodt, Phys. Rev. B 46, 6199 (1992).

${ }^{53}$ Vicinal $\operatorname{Pd}(110)$ substrate purchased from MATEK Company, Jülich, Germany. 
${ }^{54}$ A. Schatz and W. Keune, Surf. Sci. 440, L841 (1999).

${ }^{55}$ W. Kiauka, C. van Cuyck, and W. Keune, Mater. Sci. Eng., B 12, 273 (1992).

${ }^{56}$ J. Weckesser, C. Cepek, R. Fasel, J. V. Barth, F. Baumberger, T. Greber, and K. Kern, J. Chem. Phys. 115, 9001 (2001).

${ }^{57}$ W. Moritz and D. Wolf, Surf. Sci. 163, L655 (1985).

${ }^{58}$ C. M. Chan and M. A. van Hove, Surf. Sci. 171, 226 (1986).

${ }^{59}$ M. Copel and T. Gustafsson, Phys. Rev. Lett. 57, 723 (1986).

${ }^{60}$ P. Fery, W. Moritz, and D. Wolf, Phys. Rev. B 38, 7275 (1988).

${ }^{61}$ D. Tomanek, S. Wilke, and M. Scheffler, Phys. Rev. Lett. 79, 1329 (1997).

${ }^{62}$ S. L. Zhang, K. Sumiyama, and Y. Nakamura, J. Magn. Magn. Mater. 73, 58 (1988).

${ }^{63}$ E. F. Wassermann, in Magnetismus von Festkoerpern und Grenzflächen (Vorlesungsmanuskripte, IFF-Ferienkurs, Forschungszentrum Jülich, Jülich, Germany, 1993), p. 39.1 (in German).

${ }^{64}$ G. K. Wertheim, Mössbauer Effect: Principles and Applications (Academic Press, New York, 1964).

${ }^{65}$ R. D. Ellerbrock, A. Fuest, A. Schatz, W. Keune, and R. A. Brand, Phys. Rev. Lett. 74, 3053 (1995).

${ }^{66}$ W. Keune, A. Schatz, R. D. Ellerbrock, A. Fuest, K. Wilmers, and R. A. Brand, J. Appl. Phys. 79, 4265 (1996).

${ }^{67}$ B. Roldan Cuenya, M. Doi, T. Ruckert, W. Keune, and T. Steffl, J. Phys. Soc. Jpn. 69, 125 (2000).

${ }^{68}$ B. Scholz, Ph.D. thesis, Gerhard-Mercator University Duisburg, Duisburg, Germany, 1996.

${ }^{69}$ A. R. Miedema and F. Van der Woude, Physica B \& C 100B, 145
(1980).

${ }^{70} \mathrm{Ph}$. Dufek, P. Blaha, and K. Schwarz, Phys. Rev. Lett. 75, 3545 (1995).

${ }^{71}$ B. Scholz, R. A. Brand, and W. Keune, Phys. Rev. B 50, 2537 (1994).

${ }^{72}$ P. C. M. Gubbens, J. H. F. Apeldorn, A. M. van der Kraan, and K. H. J. Buschow, J. Phys. F: Met. Phys. 4, 921 (1974).

${ }^{73}$ E. E. Fullerton, D. Stoeffler, K. Ounadjela, B. Heinrich, Z. Celinski, and J. A. C. Bland, Phys. Rev. B 51, 6364 (1995).

${ }^{74}$ H. P. J. Wijn, Magnetic Properties of Metals (Springer, Berlin, 1991).

${ }^{75}$ T. C. Gibb, Principles of Mössbauer Spectroscopy (ChapmanHall, London, 1976), Chap. 8, p. 182.

${ }^{76}$ F. Van der Woude and G. A. Sawatzky, Phys. Rep., Phys. Lett. 12C, 335 (1974).

${ }^{77}$ S. Klimars, J. Hesse, and B. Huck, J. Magn. Magn. Mater. 51, 183 (1985)

${ }^{78}$ V. A. Tsurin, E. E. Yurchikov, and A. Z. Menshikov, Sov. Phys. Solid State 17, 1942 (1976).

${ }^{79} \mathrm{~S}$. Handschuh and S. Blügel (private communication).

${ }^{80}$ G. Kisters, Ch. Sauer, E. Tsymbal, and W. Zinn, Hyperfine Interact. 92, 1285 (1994).

${ }^{81}$ S. Ohnishi, A. J. Freeman, and M. Weinert, Phys. Rev. B 28, 6741 (1983).

${ }^{82}$ S. Ohnishi, M. Weinert, and A. J. Freeman, Phys. Rev. B 30, 36 (1984). 